$\$$ Research Square

\title{
A Data Driven Approach to Assess Complex Colour Profiles in Plant Tissues
}

Peter Andrew McAtee ( $\square$ peter.mcatee@plantandfood.co.nz )

New Zealand Institute for Plant and Food Research Ltd https://orcid.org/0000-0003-1569-6680

\section{Simona Nardozza}

New Zealand Institute for Plant and Food Research Ltd

Annette Richardson

New Zealand Institute for Plant and Food Research Ltd

Mark Wohlers

New Zealand Institute for Plant and Food Research Ltd

Robert Schaffer

New Zealand Institute for Plant and Food Research Ltd

\section{Methodology}

Keywords: computer vision, plant, fruit, colour, colour analysis, quantification of colour, region-grow

Posted Date: November 17th, 2021

DOI: https://doi.org/10.21203/rs.3.rs-1006490/v1

License: (c) (i) This work is licensed under a Creative Commons Attribution 4.0 International License.

Read Full License 


\section{Abstract}

\section{Background}

The ability to quantify the colour of fruit is extremely important for a number of applied fields including plant breeding, postharvest assessment, and consumer quality assessment. Fruit and other plant organs display highly complex colour patterning. This complexity makes it challenging to compare and contrast colours in an accurate and time efficient manner. Multiple methodologies exist that attempt to digitally quantify colour in complex images but these either require a priori knowledge to assign colours to a particular bin, or average the colours present within an assayed region into a single colour value. As such, to date there are no published methodologies that assess colour patterning using a data driven approach.

Results

In this study we present a methodology to acquire and process digital images of biological samples that contain complex colour gradients. The CIE (Commission internationale de l'éclairage / International Commission on Illumination) $\Delta E 2000$ formula was used to determine the perceptually unique colours (PUC) within images of fruit containing complex colour gradients. This process, on average, resulted in a $98 \%$ reduction in colour values from the number of unique colours (UC) in the original image. This data driven procedure summarised the colour data values while maintaining a linear relationship with the normalised colour complexity contained in the total image. A weighted $\Delta \mathrm{E} 2000$ distance metric was used to generate a distance matrix and facilitated clustering of summarised colour data.

\section{Conclusions}

Clustering showed that our data driven methodology has the ability to group these complex images into their respective binomial families while maintaining the ability to detect subtle colour differences. This methodology was also able to differentiate closely related images. We provide a high quality set of complex biological images that span the visual spectrum that can be used in future colorimetric research to benchmark method development.

\section{Background}

Due to its economic, health, and cultural significance, colour in plants has been studied for over 100 years [1]. Plant organs show a huge diversity of colours, with some organs such as flowers and fruit often displaying complex colour patterns. Different colours often extend across the tissue, cellular, and subcellular levels of the plant organ $[2,3,4,5]$. Plants control the expression of colour through the accumulation of pigments (biochromes) that are often located within the organelles of the cells $[6,7]$. They can also control colour by manipulating the $\mathrm{pH}$ of the intracellular space containing the pigment and through the organisation of pigment containing cell layers $[2,8,9]$. Fruit often display a wide range of colour patterning in their external tissues, with differences observed between the exposed blush and shaded non-blush sides of fruit $[8,10]$ as well as between the external and internal tissues of an organ 
[11]. Colour patterning can also vary between different species of the same genus and even between different cultivars of the same species $[7,12,13,14,15,16]$.

In a biological context, colour can be used by plants as a signalling mechanism to other organisms that might interact with the plant $[17,18,19]$. These interactions can include the inhibition of predation of leaves, the attraction of pollinators to flowers, or in the case of fruit, as a cue to potential seed dispersers that the fruit is ready to be consumed. In addition to displaying colouration differences between organs, some plants are also capable of changing the colour of an organ during development and/or in response to an environmental cue $[8,9,20]$. The diverse range of colour polymorphism observed in the plant kingdom is particularly evident in flowers and fruit but is also present in the stems, leaves, tubers, and roots.

It is still common for academic and industrial studies to grade the colour of plant organs using subjective visual scales involving human participants. This is a challenging task to achieve in a precise or objective manner due to the complex nature of colour patterns observed in plant organs like fruit [21]. There are also significant analytical challenges when comparing human observed colour data due to variation between individual participants in both the type and the number of photoreceptors used to detect colour [22]. Other factors that can influence the perception of colour by humans include recall memory, retinal fatigue, and interactive effects of the background and shape of the object [23, 24, 25]. These factors make it difficult to objectively compare human perceived colour data with simple patterning. The complex and multifaceted nature of plant colour composition makes accurate description of human perceived colour data largely impossible.

Multiple research methodologies have been employed to circumvent the human perception of colour [10]. Some studies have aimed to directly measure the chemical composition of plant biochromes within the cells of sampled tissue. These studies attempted to use the quantified chemical compound(s) as a proxy to estimate the colour. This methodology assumes linearity between the quantity of the chemical and physiological presentation of the colour and does not account for the 2-dimensional or 3-dimensional distribution of biochromes within the plant organ. Advances in digital technologies have offered an alternative approach to quantify and compare colour attributes. Some commonly used tools include the Konica Minolta Chroma-meter that digitally captures the reflectance of light within a known radial area. These tools output a device-independent trinary CIE $1976\left(\mathrm{~L} * a * b^{\star}\right)$ coordinate that describes the colour of the object by averaging the reflectance values within the measured area $[26,27,28]$. This type of colour measuring system is suitable when assessing an object with a high degree of uniformity in colour but is less useful when trying to assess the complex colour patterning observed in fruit.

Most commercially available image capture devices output data in the device-dependent RGB colour space. The RGB colour space contains more than 16 million colours making any comparison on a colourby-colour basis between samples prohibitively long in terms of computational time $[27,29,30]$. An additional challenge for these types of sensors is the incident light source itself can affect the perception of colour (reflected light). Fortunately this can be compensated for by including an internal colour 
standard within the context of the acquired image and by controlling the ambient light source using a controlled environment (imaging-box) [31].

Currently, multiple methodologies exist that attempt to digitally quantify colour in images but these either require a-priori knowledge to assign colours to a particular bin [32,33], or average the colours present within a assayed region into a single colour value [34]. In this study we developed a standardised method to measure colour and a data driven methodology to summarise and quantify different colour patterning in cross sectional fruit/tuber images, greatly simplifying the complexity of colours identified in the images. The colour data simplification using this novel methodology were used to rebuild virtual images as a validation process.

\section{Results}

\section{Scanner based imaging}

To standardise the colour space measurements, a flatbed scanner was used within a purpose built lightproof box. This setup was developed as it provided a consistent light source that was unaffected by changes in the ambient light. The box was of sufficient depth to allow space for a thick layer of plant tissue to be placed on the scanner without admitting any light through the sample. Using this setup, the cross sections of 28 diverse fruit and tubers were scanned and stored as different compression formats for analysis.

\section{Assessment of image formats}

Two different image file formats were compared: lossless (TIFF) and lossy (JPEG). A pixel-for-pixel comparison showed that JPEG compression had a significant effect on the colour values. This compression effect varied between different images. The lowest and highest mean mismatch percentages were observed in avocado and tamarillo images with mean mismatch percentages of $2.17 \%$ and $29.40 \%$ respectively (Table 1 ). The effect of the JPEG compression algorithm on mismatch frequency increased with increasing colour complexity of the fruit, however, this relationship was not linear (Figure 1). To identify the location at which colour differences occurred in these image formats, three representative images where selected according to their low mismatch (Pear Green R1), mid-range mismatch (Lemon R1), and high mismatch (Kiwifruit Red R3) frequency. The two dimensional distribution of mismatches in JPEG images tended to occur in regions of the fruit where the colour gradient changed (Figure 2). In all images, there was a higher amount of mismatches at the intersection of the background and foreground. No predictable pattern of mismatches was observed within each subject image. 
Table 1

A comparison between the JPEG and PNG file formats. This table summarises the number of pixels that were identical, similar, or different in each comparison. Data are presented as mean \pm standard error and represent three biological replicates for each class of fruit/tuber with the exception of mango for which only one fruit was sampled.

\begin{tabular}{|c|c|c|c|c|}
\hline Subject type & Identical in JPEG & Similar in JPEG & Different in JPEG & Identical in PNG \\
\hline Apple 'Braeburn' & $2.42 \pm 0.05$ & $89.17 \pm 0.05$ & $8.42 \pm 0.61$ & 100 \\
\hline Apple 'Granny Smith' & $1.42 \pm 0.08$ & $93.44 \pm 0.08$ & $5.14 \pm 0.43$ & 100 \\
\hline Apple 'Pacific Rose' & $2.11 \pm 0.16$ & $92.88 \pm 0.16$ & $5.01 \pm 0.46$ & 100 \\
\hline Apple 'Royal Gala' & $2.36 \pm 0.30$ & $91.70 \pm 0.3$ & $5.94 \pm 1.18$ & 100 \\
\hline Avocado & $3.50 \pm 0.09$ & $94.33 \pm 0.09$ & $2.17 \pm 0.42$ & 100 \\
\hline Banana & $2.68 \pm 0.19$ & $90.43 \pm 0.19$ & $6.89 \pm 0.70$ & 100 \\
\hline Grapefruit & $1.68 \pm 0.14$ & $90.11 \pm 0.14$ & $8.21 \pm 0.59$ & 100 \\
\hline Kiwifruit Gold & $1.05 \pm 0.02$ & $79.61 \pm 0.02$ & $19.34 \pm 0.79$ & 100 \\
\hline Kiwifruit Green & $1.18 \pm 0.07$ & $80.86 \pm 0.07$ & $17.97 \pm 0.41$ & 100 \\
\hline Kiwifruit Red & $0.57 \pm 0.02$ & $70.98 \pm 0.02$ & $28.45 \pm 0.39$ & 100 \\
\hline Kumara Gold & $2.15 \pm 0.05$ & $92.42 \pm 0.05$ & $5.43 \pm 0.36$ & 100 \\
\hline Kumara Red & $5.98 \pm 1.09$ & $90.86 \pm 1.09$ & $3.17 \pm 0.24$ & 100 \\
\hline Lemon & $2.31 \pm 0.26$ & $87.74 \pm 0.26$ & $9.95 \pm 1.63$ & 100 \\
\hline Mandarin & $0.97 \pm 0.05$ & $86.77 \pm 0.05$ & $12.26 \pm 0.66$ & 100 \\
\hline Mango & $1.71 \pm N A$ & $94.16 \pm N A$ & $4.13 \pm N A$ & 100 \\
\hline Orange & $1.44 \pm 0.11$ & $88.79 \pm 0.11$ & $9.78 \pm 0.71$ & 100 \\
\hline Peach & $1.92 \pm 0.06$ & $92.69 \pm 0.06$ & $5.40 \pm 0.22$ & 100 \\
\hline Pear 'Corella' Brown & $2.72 \pm 0.21$ & $91.48 \pm 0.21$ & $5.81 \pm 0.08$ & 100 \\
\hline Pear Green & $3.39 \pm 0.03$ & $89.89 \pm 0.03$ & $6.72 \pm 0.46$ & 100 \\
\hline Pear Nashi Brown & $1.53 \pm 0.04$ & $84.9 \pm 0.04$ & $13.57 \pm 0.49$ & 100 \\
\hline Persimmon & $1.03 \pm 0.04$ & $92.17 \pm 0.04$ & $6.8 \pm 0.07$ & 100 \\
\hline Plum & $1.43 \pm 0.18$ & $81.81 \pm 0.18$ & $16.76 \pm 2.21$ & 100 \\
\hline Potato Purple & $1.21 \pm 0.12$ & $86.6 \pm 0.12$ & $12.19 \pm 1.12$ & 100 \\
\hline
\end{tabular}

Supplementary Figure 1: Side-by-side comparison of avocado images recoloured with data processed with region-growing or k-means methodologies. The textural differences in colour are most pronounced between the k-means and the original image. 


\begin{tabular}{|lllll|}
\hline Subject type & Identical in JPEG & Similar in JPEG & Different in JPEG & Identical in PNG \\
\hline Potato Red & $2.25 \pm 0.33$ & $92.48 \pm 0.33$ & $5.27 \pm 1.44$ & 100 \\
\hline Potato White & $1.88 \pm 0.1$ & $89.08 \pm 0.1$ & $9.04 \pm 0.88$ & 100 \\
\hline Strawberry & $0.57 \pm 0.05$ & $80.32 \pm 0.05$ & $19.11 \pm 1.55$ & 100 \\
\hline Tamarillo & $0.67 \pm 0.04$ & $69.94 \pm 0.04$ & $29.39 \pm 1.36$ & 100 \\
\hline Tomato & $0.86 \pm 0.03$ & $80.42 \pm 0.03$ & $18.72 \pm 0.41$ & 100 \\
\hline $\begin{array}{l}\text { Supplementary Figure 1: Side-by-side comparison of avocado images recoloured with data processed } \\
\text { with region-growing or k-means methodologies. The textural differences in colour are most } \\
\text { pronounced between the k-means and the original image. }\end{array}$ & \\
\hline
\end{tabular}

\section{Colorimetric analysis of fruit/tuber images}

To assess colour variability in the images, the colour values of each pixel from the TIFF format images were transcoded to hexadecimal code. Frequency tables of the hexadecimal codes were then constructed to determine the number of unique colour (UC) values in each subject image (Supplementary Table 1). The number of UC values within each subject imaged was highly variable among the fruit analysed in this study. The lowest number of UC values was observed in Potato White R3, with 34,629 values and the highest number of UC values was observed in Mandarin R2, with 202,377 values. Ordered frequency distributions demonstrated that the majority of hexadecimal codes had a frequency of less than 10 values across all of the subject images used in this study (Figure 3). This demonstrated that there are a large number of subtle colour differences within the plant tissues.

To simplify the colour space data, a region-growing algorithm was developed to condense the colour descriptors within each subject image into perceptually unique colours (PUC) in an unbiased manner. The algorithm reduced the density of colorimetric data points on average by $98 \%$. The highest number of colour values after the region-growing algorithm was applied, using a threshold $\Delta E 2000$ value of 2 , was 3078 (Supplementary Table 1). Applying an additional threshold to the summarised region-grown colour values that contributed $>0.1 \%$ of the total percentage of each image reduced the data density on average by $99.9 \%$ with a maximum of 219 colour values. The biggest determinant of the processing time was the number of UC values that each raw image contained (Supplementary Table 2).

To determine the impact on the overall processing time and compression of the data, the $\Delta E 2000$ threshold of the region-growing algorithm was tested at 1, 1.5, 2, 2.5, and 3 (Supplementary Table 2). Relative to a $\triangle \mathrm{E} 2000$ threshold of 2 , increasing the threshold to 2.5 and 3 reduced processing time on average by a ratio of 0.86 and 0.71 , respectively. In terms of the number of output colour bins, increasing the $\Delta \mathrm{E} 2000$ threshold to 2.5 and 3 reduced the average number of colour bins by a ratio 0.59 and 0.38 , respectively, relative to a threshold of 2 . Relative to a $\Delta \mathrm{E} 2000$ threshold of 2 , decreasing the $\Delta \mathrm{E} 2000$ threshold to 1.5 and 1 increased processing time 1.5 and 2.9 fold, respectively. This also increased the number of output colour bins by a ratio of 2 and 4.6 , respectively. 


\section{Comparison with K-means clustering}

The region-growing methodology was compared with the widely used k-means clustering methodology in order to assess its processing efficiency. The region-growing algorithm (at $\Delta \mathrm{E} 2000$ threshold $=2$ ) was faster than k-means clustering using a K-value of 20 or 100, with ratios in processing time of 0.95 and 0.79 respectively (Supplementary Table 2 ).

To back validate the efficiency of the colour data clustering generated from the region-growing and kmeans cluster analysis, the outputs of these algorithms were used to raster new images as described in the Methods section. An example of this process can be seen in Figure 4, which shows that there was a high degree of visual similarity between the original image and the images generated using output colour bins generated at $\triangle \mathrm{E} 2000$ thresholds of 2 and 3 . Inspection of the k-means-generated images showed considerable loss of colour texture within each image (Figure 4D and Supplementary Figure 1). This was particularly evident in the seeds of the apple, around the skin of the lemon, and at the central core of the kiwifruit.

Image compression value was used to determine how well the original image complexity was represented by the region grown and $\mathrm{k}$-means clustered algorithms. The region-growing algorithm resulted in linear regression values of $0.90,0.84,0.80,0.76$, and 0.72 for $\Delta \mathrm{E} 2000$ thresholds of $1,1.5,2,2.5$, and 3 , respectively. The k-means clustering algorithm resulted in linear regression values of 0.71 and 0.71 at $\mathrm{K}$ values of 20 and 100, respectively (Supplementary Table 2).

\section{Clustering of region-grown data across a broad spectrum of colours}

The weighted $\Delta \mathrm{E} 2000$ distance metric was used to generate a distance matrix using the region-grown colour output for each image. Hierarchical clustering of the weighted distance matrix was used to cluster the data into 10 groups (Figure 5). A dendrogram, with the respective images aligned, shows that each of the 10 clusters was associated with a different colour attribute. For example, the 'lighter' orange hue associated within Cluster 5 grouped orange, mango, and grapefruit together and this was different from the 'darker' orange hue that separated mandarin into Cluster 4. In general, all fruit types were co-localised with the exception of Tamarillo R2 and Potato Red R3. Visual inspection of the Tamarillo R2 image showed that is contained a greater amount of 'lighter' orange hues compared to the other tamarillo replicate images. Visual inspection of the Potato Red R3 showed that it contained a greater amount of the yellow/orange colour hues compared with its replicate images. In both these cases the outlier images were visually distinct from their respective replicates, which demonstrates the need for replicates when undertaking colour studies.

\section{Fruit with different colour patterns}

To extend the utility of this methodology into fruit with similar but complex patterns, a larger experiment using 100 red fleshed kiwifruit Actinidia chinensis var. chinesis ('Zes008') was undertaken. Red fleshed 
kiwifruit often have an intense inner pericarp red colour and a lighter outer pericarp colour [35]. In other species such as apple, exclusion of environmental light is known to increase red pigment (anthocyanin) accumulation in the skin, though less is known about the effect of light exclusion on internal fruit tissues. To test if exclusion of environmental light affects the internal fruit tissues of kiwifruit, a total of 50 fruit were bagged to induce red colour accumulation while 50 control fruit were left unbagged.

In order to differentiate the colour values located in the outer and inner pericarp of kiwifruit images, a script was developed to automatically identify the boundaries of these tissue zones and segment the inner and outer pericarp tissue regions of fruit. This allowed the lighter coloured outer pericarp to be separated from the darker red of the inner pericarp (Figure 6A).

These segmented images were then summarised using the region-growing algorithm and clustered using the weighted $\triangle E 2000$ metric. To assess variability within the condensed colour space (PUC) profiles the inner and outer pericarp tissues were K-means clustered. The outer pericarp showed greater variability in colour profiles (maximum height of the dendrogram was 268.63) compared with the inner pericarp (maximum height of 141.46) (Figure 6A and B). Surprisingly, the outer pericarp clusters 2, 3, and 6 (associated with bagged fruit) contained greater amounts of red in their colour profiles. The outer pericarp colour profiles of unbagged fruit were largely localised in clusters 1, 5, and 8 and were associated with more yellow/green colour profiles.

\section{Discussion}

The analysis of colour patterning of complex images like fruit can be a time consuming and subjective process. Our approach aimed to develop a data driven and time efficient methodology that could summarise the diverse colour patterning observed in nature by clustering colour values that have no perceptible difference from each other. In this study we selected fruit types to encompass a wide variety of colours across the visible spectrum. The amount of unique colours within the images used in this study ranged from tens of thousands to hundreds of thousands. The relatively high density of colour data points within each image made it challenging to summarise the data to an amount that was unbiased and still meaningful to human interpretation. The CIE $\Delta E 2000$ formula provided a reliable basis to measure the perceptual distance between colours in the $L * a * b *$ colour space [36, 37]. The formula was used in this study to measure generalised colour complexity and formed the basis for developing the region-growing algorithm. $\Delta \mathrm{E} 2000$ values below 2 are regarded as having minimal perceptual difference by the human eye while values greater than 2 but less than 3 are regarded as being distinguishable by the human eye at a glance $[38,39]$. These are, however, generalised rules and it should be noted that there is a wide range of variation in the perceptive ability of individuals [22].

K-means clustering or segmentation of the colour gamut into predefined bins are two of the most common approaches to speed up the processing time of digital colorimetric analysis of images $[39,40$, $41,42]$. These methodologies output arrays in regular formats that are easy to compare, but have the drawback of requiring a-priori knowledge of a predefined number of bins to which to assign each colour. 
Additionally, both of these approaches describe the colour value for a given bin as an average value that might not exist within the context of the original dataset. The region-growing approach developed in this study solves this issue by using a data driven approach to independently interrogate and grow the optimal number of colour bins for each image. This data driven approach is unbiased but has the drawback of having an irregular output array. The weighted $\Delta \mathrm{E} 2000$ distance metric is a robust method that allowed us to measure the distance between the irregular output arrays (colour profiles) produced by the region-growing algorithm. The concept of this metric was based on the Earth Movers Distance (EMD) metric which is commonly used to measure differences between colour profiles $[43,44,45,46]$. EMD uses a Euclidean distance metric which poorly approximates the colour space a human perceives. The EMDlike process (weighted $\triangle E 2000$ distance metric) developed in this publication instead uses the $\Delta E 2000$ formula to calculate distance. Our analysis demonstrates the utility of this mathematical method to cluster images based on their region-grown outputs. We were able to cluster images while maintaining the ability to detect subtle colour differences that are commonly observed in biological samples such as fruit. This can be most notably observed in the dissimilar cluster localisation of Potato Red R2 and Tamarillo R2 images outside of the clusters containing their respective replicates and is further demonstrated by the comparison of fruit with close visual similarity shown in Figure 6 . We observed a stronger regression score for images generated using the region-grown methodology than for those generated via k-means clustering, when compared with the compression rate of the original image. This indicates that colour value outputs of the region-growing methodology better approximated those observed in the original image than did outputs generated by the k-means analysis. Additionally, visual inspection of the region-grown in-silico simulated images showed more consistency in their visual similarity to the original than those generated by k-means clustering. This is perhaps unsurprising as the k-means clustering approach is often subjected to under or over fitting on data, particularly when the overall complexity of the data is not known in advance, as is the case in biological images.

This study aimed to develop a data driven approach for quantifying colour. Understanding the impact of image file format on the data was an important. Interestingly, there is relatively little published research that quantifies the effect that image data compression algorithms have on the colour values of an image. The two types of data compression algorithms that are used by image acquisition tools are lossless (TIF and PNG) and lossy (JPEG). Lossless image compression has the ability to restore the data to its original value after decompression but produces relatively large image files with higher storage overheads [47]. Lossy compression does not allow full restoration of the original data values but produces much smaller image files, thereby reducing storage overheads [47]. Selecting the most suitable image format is the first decision step in an image analysis pipeline and ultimately can influence the downstream analysis of the image. Here we compared three image formats (TIF, PNG, and JPEG) to quantify the effect of different data compression algorithms on colour values from complex biological images, and showed that uncompressed files are the only way to maintain the complexity of data in fruit images. While the small file sizes produced by JPEG compression are attractive from a perspective of file storage, this compression affects the integrity of the data and is likely to have implications for applications that require precision colour estimation including machine learning applications. 


\section{Conclusions}

This publication demonstrates the utility of a data driven approach for the analysis of images with complex colour profiles. We first developed a novel algorithm to reduce the density of data within each image in a process called region growing. The processing time of this algorithm was comparable with other commonly used methodologies such as K-means clustering. One major advantage of our approach is that it does not require a-priori knowledge of the amount of $\mathrm{K}$-mean bins to generate. This is important for the comparison of images based on their colour features as it avoids subjective under/over fitting of data. Central to our methodology was the use of the previously published CIE $\triangle \mathrm{E} 2000$ formula. This formula was used to quantify the difference between colours in a colour-space that aligns with human perceptive ability. This is different from other methodologies that use euclidean distances to measure differences in colour data.

We also developed a novel process to compare the region-grown colour profiles from a dataset with diverse colour patterning and from a separate dataset with visually similar colour patterning. The utility of this methodology could have significant implications towards improving the accuracy of computer vision phenotyping of plant tissues particularly in regard to colourimetric analysis. Applications of this methodology could extend across various plant related academic and research disciplines including plant breeding, and postharvest quality assessment.

\section{Methods}

\section{Image capture}

A Canon LIDE 220 flatbed scanner was placed in a $2 \mathrm{~mm}$ black perspex box with a retractable lid to completely block ambient light. A transparent A4 sized sheet with a printed $3 \times 1$ grid $(140 \mathrm{~mm} \times 95 \mathrm{~mm})$ was placed on the scanner platen along with an internal colour standard in the form of an X-Rite minicolour checker card [48]. An A4 sized image at a resolution of 600DPI was output in a TIF, PNG, and JPEG file format.

A diverse selection of 28 species of fruit and tubers was purchased from a local supermarket in Auckland, New Zealand. These fruit and tubers represented different families including Anacardiaceae (mango), Ebenaceae (persimmon), Actinidiaceae (kiwifruit), Lauraceae (avocado), Musaceae (banana), Rosaceae (apple, peach, pear, plum, and strawberry), Rutaceae (grapefruit, lemon, mandarin, and orange), and Solanaceae (potato, tamarillo, and tomato). Each fruit was cross sectioned along its most symmetrical side. Up to three cross sections of the same fruit type were placed face down on the scanner on a predefined $3 \times 1$ grid with defined positions to allow image capture of the individual fruit.

In a more detailed experiment to evaluate the effect of light exclusion on anthocyanin production in fruit flesh, 100 Actinidia chinensis var. chinensis 'Zes008' (red-fleshed kiwifruit) were also studied. These fruit were harvested from a commercial orchard based in Kerikeri New Zealand (for details see [49]). During 
fruit growth these fruit were either bagged with lightproof bags [50] from 56 days after anthesis (DAA) for 108 days or left unbagged (control).

\section{Colour standardisation and segmentation}

The flatbed scanner used in this study used the device dependent RGB colour space to denote colour value. It is well established practice that images are standardised to a control even in controlled lighting conditions for differences in sensor sensitivity. Fiji/ImageJ (version 1.52p) [51] was used to calibrate the colour images using the white tile standard on the X-Rite mini-colour checker card that was included in each scanned image. Each fruit was then segmented from its parent image using a colour thresholding method that excluded the background colour (black) and saved as an independent sibling image in TIF, JPEG, or PNG file format

\section{Colorimetric analysis}

Analysis of colour data values was done on sibling images segmented from the background using the open source software R (v3.6.1) [52]. Two colour space transcoding processes were used in this study. A transcode between the gamut of RGB colour values in each image to the hexadecimal syntax was done using the $r g b$ function supplied by the grDevices (v3.6.2) package [52]. A transcode between the gamut of RGB colour values into the CIE $1976\left(\mathrm{~L}^{*} \mathrm{a} \mathrm{b}^{*}\right)$ space was achieved using the convertColor function tools supplied as part of the grDevices (v3.6.2) package.

The $\triangle \mathrm{E} 2000$ distance measure was calculated using the CIE 1976 ( ${ }^{*}{ }^{*}{ }^{*} \mathrm{~b}^{\star}$ ) transcoded colour data and the deltaE2000 function supplied as part of the colorscience (v1.0.8) package [53]. This equation was used to group colour values based on similarity using the region-growing algorithm, quantify differences/similarities between pixel-for-pixel comparisons of colour values between file formats, and to cluster images using the weighted CIE $\Delta \mathrm{E} 2000$ distance metric.

Tools supplied as part of the EBImage package were used to parse, modify, and save image files [54]. The circlize package was used to generate the circular dendrograms presented in Figure 5 and Figure 6 [55].

The region-growing algorithm was developed and implemented in $\mathrm{R}$ version 3.6. This algorithm begins by prioritising the most frequent unique colour (UC) values within an image. The most frequent UC value is set as a seed and it is recursively tested against all the other UC values (queries) using the CIE $\triangle \mathrm{E} 2000$ formula. If the result of this calculation is below a threshold value $(<=2)$ then the frequency of the query value is reassigned to the seed value and the query value is removed from the pool. This process repeats until all the UC values have been tested and/or reassigned. The final output is a list of perceptually unique colours (PUC) and their frequencies (as a percentage of the total image) known as a PUC-table. The pseudocode for the process is outlined below.

Declare double: $d, F_{s N o r m}$

Declare vector (character): vec_hex 
Declare vector (double): vec_freq

Declare $L^{*} a^{*} b^{\star}$ vector (double): $L_{q}, L_{t}$

Declare character: $H_{s} H_{q}$

Declare integer $n, F_{t} F_{s}, F_{q}$

1. Covert the RGB pixel values to a vector of hexadecimal values

2. Generate a frequency table of unique hexadecimal codes (vec_hex and vec_freq)

3. Rank vec_hex and vec_freq in descending order of frequency

vec_hex and vec_freq now contain corresponding ordered hexadecimal codes and frequencies

1. Set $f_{t}=$ sum of all frequencies

2. Set $n_{s}=$ number of hexadecimal codes

3. Set $n_{q}=$ number of hexadecimal codes

4. Loop: Repeat for less than or equal to $n_{s}$

Loop: Repeat for less than or equal to $n_{q}$

Set $d=0$

Set $f=0$

Set $H_{s}=v e c \_h e x\left[n_{s}\right]($ seed hexadecimal code)

Set $H_{q}=v e c \_h e x\left[n_{q}\right]$ (query hexadecimal code)

Set $F_{s}=v e c_{-}$freq $\left[n_{s}\right]$ (seed frequency)

Set $F_{q}=$ vec_freq $\left[n_{q}\right]$ (query frequency)

Covert $H_{s}$ and $H_{q}$ to $L * a * b *$ colour space $\left(L_{s}\right.$ and $\left.L_{q}\right)$

Calculate $d$ using $\Delta \mathrm{E} 2000$ equation between $L_{s}$ and $L_{q}$

if $d<=2$ then

Calculate $F_{s}=F_{s}+F_{q}$

Remove vec_hex $=v e c \_h e x\left[-n_{q}\right]$

Remove vec_freq $=$ vec_freq $\left[-n_{q}\right]$ 
End loop

Calculate $F_{s N o r m}=F_{S} / F_{t}^{*} 100$

Write to output $H_{s}$ and $F_{s N o r m}$ as Region Grow data

End loop

Recolouring of images was also done using $\mathrm{R}$ version 3.6 and the EBImage package. This process uses the region-growing algorithm stated above. It tests the colour values of all the pixels in an image and reassigns the colour value of a pixel if the CIE $\triangle E 2000$ distance (relative to a tested seed colour) is below the defined threshold value. In this manner all the colours in an image are reassigned the value of their perceptually unique bin and a perceptually unique raster of the image is made. The following pseudocode was used to recolour images.

Declare double: $d$

Declare $L^{*} a * b^{*}$ array (double): $L_{R G} L_{\text {lmage }}$

Declare $L^{*} a^{*} b^{*}$ vector (double): $L_{q}, L_{t}$

Declare integer $n_{i} n_{r}$

1. Open Region Grow data and corresponding Image data

2. Covert Region Grow data hexadecimal code to $L^{*} a * b *$ colour space $\left(L_{R G}\right)$.

3. Covert Image data hexadecimal code to $L^{*} a b^{*}$ colour space $\left(L_{\text {Image }}\right)$.

4 . Set $n_{r}=$ number of row values in $L_{R G}$ (Region grow data)

5. Set $n_{i}=$ number of pixels in $L_{\text {Image }}$ (Image data)

6. Loop: Repeat for less than or equal to $n_{r}$

Loop: Repeat for less than or equal to $n_{i}$

Set $d=0$

Set $L_{s}=L_{R G}\left[n_{r}\right]$ (seed)

Set $L_{q}=L_{\text {lmage }}\left[n_{j}\right]$ (query)

Calculate $d$ using $\Delta \mathrm{E} 2000$ equation between $L_{s}$ and $L_{q}$

if $d<=2$ then

Assign $L_{s}$ value to $L_{\text {Image }}\left[n_{i}\right]$ 
Remove $L_{\text {lmage }}=L_{\text {Image }}\left[-n_{j}\right]$ from pool of values to test

End loop

End loop

1. Raster image and save

The weighted CIE $\triangle \mathrm{E} 2000$ distance metric was developed and implemented in $\mathrm{R}$ version 3.6. This metric works by using the 'perceptually unique colour (PUC) tables' generated by the region-growing algorithm. A 'transport matrix' is constructed by undertaking a full-rank comparison of all of the PUC values contained between two PUC-tables (images). The CIE $\triangle E 2000$ distance function is used to calculate a distance metric between each respective colour comparison. A 'weighed distance value' is then generated by multiplying the $\triangle E 2000$ distance value by the difference in frequency (as a percentage) of the respective PUC comparison. The closest match to a PUC value is considered as the minimum weighed distance value contained in each row of the 'transport matrix'. The overall similarity between two PUC profiles (all the values in a PUC-table) can then be considered as the sum of the minimum weighed distance values (closest matches). This is similar to Wasserstein metric but uses the CIE $\triangle E 2000$ formula to define distance and factors in the frequency/abundance difference between colour values. The following pseudocode was used to generate the CIE $\triangle \mathrm{E} 2000$ weighted distance matrix.

Declare double: $d$, Freq $_{q}$, Freq ${ }_{t}$, Freq $_{\text {Diff }}$

Declare integer: $q, t, n r_{q}, n r_{t}$

Declare $L^{*} a^{*} b^{\star}$ array (double): $L_{\text {Query }} L_{\text {Target }}$

Declare $L{ }^{*} a{ }^{*}{ }^{*}$ vector (double): $L_{s}, L_{t}$

Declare array (double): Query_array, Target_array

Declare array (double): Transport_array $\left[n_{q}, n_{t}\right]$

Declare vector (double): vector_min $\left[n_{q}\right]$

Declare double: cumulative sum

Declare array (double): Distance_array $[q, t]$

Nested Loop:

1. Loop: For $q$ in number of Region Grow data tables

Loop: For $t$ in number of Region Grow data tables 
Set Query_array = Region Grow data table[q]

Set Target_array $=$ Open Region Grow data table[t]

Set $n r_{q}=$ row dimension of Query_array

Set $n r_{t}=$ row dimension of Target_array

Set $L_{\text {Query }}=$ Covert Query_array hexadecimal codes to $L^{*} a * b^{\star}$ colour space

Set $L_{\text {Target }}=$ Covert Target_array hexadecimal codes to $L^{*} a * b *$ colour space

Nested Loop:

1. Loop: Repeat for less than or equal to $n r_{q}$

2. Loop: Repeat for less than or equal to $n r_{t}$,

Set $d=0$

Set $L_{q}=L_{\text {Query }}\left[n r_{q}\right]$

Set $L_{t}=L_{\text {Target }}\left[n r_{t}\right]$

Calculate $d$ using $\Delta \mathrm{E} 2000$ equation between $L_{q}$ and $L_{t}$

Set Freq $_{q}=$ Query_array $\left[n r_{q}\right]$ (Query frequency data)

Set Freq $_{t}=$ Target_array $\left[n r_{t}\right]$ (Target frequency data)

Calculate Freq $_{\text {Diff }}=$ Freq $_{q}-$ Freq $_{t}$

if $d=0$ then

Set Transport_array $\left[n_{q}, n_{t}\right]=$ Freq $_{\text {Diff }}$

else

Set Transport_array $\left[n_{q}, n_{t}\right]=d^{\star}$ Freq $_{\text {Diff }}$

End loop

End loop

Calculate vector_min = Find minimum value in each row of Transport_array

Calculate cumulative sum $=$ sum of all values in vector_min 
Set Distance_array $[q, t]=$ cumulative sum

End loop

End loop

1. Save distance matrix Distance_array

\section{Abbreviations}

$\triangle E 2000$ : A mathematical formula used for measuring the similarity of tristimulus values

CIE: Commission internationale de l'éclairage / International Commission on Illumination

PUC: Perceptually unique colours

PUC-table: A table containing the hexadecimal codes of the perceptually unique colours generated using the region-growing algorithm. Also included in this table is the corresponding percentage cover of the subject that assigned in each PUC.

RGB: Tristimulus colour channels (Red, Green, Blue)

UC: Unique colours

\section{Declarations}

Ethics approval and consent to participate

Not applicable

Consent for publication

Not applicable

Availability of data and materials

The datasets used and/or analysed during the current study are available from the corresponding author on reasonable request.

Competing interests

The authors declare that they have no competing interests

Funding 
This study was funded by PFR Kiwifruit Royalty investment fund and the large kiwifruit study was funded by Zespri project: Zespri NP1974.

Authors' contributions

RS, PM conceived the project and RS obtained funding. PM, AR, SN generated the data, PM developed the algorithm, which were checked and improved by MW. PM and RS wrote the manuscript and all authors checked and edited the final paper.

Acknowledgements

We would like to acknowledge Missy Power and the FAST lab team for collecting the kiwifruit images used in this publication.

\section{References}

1. Kraemer H. Studies on Color in Plants. Bulletin of the Torrey Botanical Club. 1906;33(2):77-92.

2. Noda K, Glover BJ, Linstead P, Martin C. Flower Color Intensity Depends on Specialized CellShape Controlled by a Myb-Related Transcription Factor. Nature. 1994;369(6482):661-4.

3. Lister CE, Lancaster JE, Walker JRL. Developmental changes in enzymes of flavonoid biosynthesis in the skins of red and green apple cultivars. J Sci Food Agr. 1996;71(3):313-20.

4. Rat'kin AV. Formation of flavonoid pigments during flower color development in sweet pea mutants. Izv Akad Nauk Biol+. 2000(5):538-45.

5. Tan JF, Wang MJ, Tu LL, Nie YC, Lin YJ, Zhang XL. The Flavonoid Pathway Regulates the Petal Colors of Cotton Flower. Plos One. 2013;8(8).

6. Mol J, Grotewold E, Koes R. How genes paint flowers and seeds. Trends Plant Sci. 1998;3(6):2127.

7. Moreau C, Ambrose MJ, Turner L, Hill L, Ellis THN, Hofer JMI. The b Gene of Pea Encodes a Defective Flavonoid 3 ',5 '-Hydroxylase, and Confers Pink Flower Color. Plant Physiol. 2012;159(2):759-68.

8. Lancaster JE. Regulation of Skin Color in Apples. Crit Rev Plant Sci. 1992;10(6):487-502.

9. Liu LH, Shao ZY, Zhang M, Wang QM. Regulation of Carotenoid Metabolism in Tomato. Mol Plant. 2015;8(1):28-39.

10. Delgado-Pelayo R, Gallardo-Guerrero L, Hornero-Mendez D. Chlorophyll and carotenoid pigments in the peel and flesh of commercial apple fruit varieties. Food Res Int. 2014;65:272-81. 
11. Espley RV, Hellens RP, Putterill J, Stevenson DE, Kutty-Amma S, Allan AC. Red colouration in apple fruit is due to the activity of the MYB transcription factor, MdMYB10. Plant J. 2007;49(3):414-27.

12. Goodrich J, Carpenter R, Coen ES. A Common Gene Regulates Pigmentation Pattern in Diverse Plant-Species. Cell. 1992;68(5):955-64.

13. De Jong WS, Eannetta NT, De Jong DM, Bodis M. Candidate gene analysis of anthocyanin pigmentation loci in the Solanaceae. Theor Appl Genet. 2004;108(3):423-32.

14. Zhao DQ, Tao J, Han CX, Ge JT. Flower color diversity revealed by differential expression of flavonoid biosynthetic genes and flavonoid accumulation in herbaceous peony (Paeonia lactiflora Pall.). Mol Biol Rep. 2012;39(12):11263-75.

15. Ilahy R, Riahi A, Hdider C, Tlili I, Dalessandro G, Lenucci MS. Carotenoids Content in Intact Plastids Isolated from Ordinary and High-Lycopene Tomato (Solanum lycopersicum L.) Cultivars. Acta Hortic. 2015;1081:135-40.

16. Iwashina T, Mizuno T. Flavonoids and Xanthones From the Genus Iris: Phytochemistry, Relationships with Flower Colors and Taxonomy, and Activities and Function. Nat Prod Commun. 2020;15(10).

17. Chittka L, Menzel R. The Evolutionary Adaptation of Flower Colors and the Insect Pollinators Color-Vision. J Comp Physiol A. 1992;171(2):171-81.

18. Clegg MT, Durbin ML. Flower color variation: A model for the experimental study of evolution. $\mathrm{P}$ Natl Acad Sci USA. 2000;97(13):7016-23.

19. Koes RE, Quattrocchio F, Mol JNM. The Flavonoid Biosynthetic-Pathway in Plants - Function and Evolution. Bioessays. 1994;16(2):123-32.

20. Shen HJ, Guo QS, Fang HL. Impact of different colored shade polyethylene and maturation on development of flavonoids and phenolic acids in flowers of Chrysanthemum indicum L. J Med Plants Res. 2011;5(29):6555-63.

21. Thierry G, Athanasopoulos P, Wiggett A, Dering B, Kuipers J. Unconscious effects of languagespecific terminology on preattentive color perception. PNAS. 2008;106(11):4567-70.

22. Nathans J, Piantanida TP, Eddy RL, Shows TB, Hogness DS. Molecular-Genetics of Inherited Variation in Human Color-Vision. Science. 1986;232(4747):203-10.

23. Bloj MG, Kersten D, Hurlbert AC. Perception of three-dimensional shape influences colour perception through mutual illumination. Nature. 1999;402(6764):877-9. 
24. Duffy VG, Chan AHS. Effects of virtual lighting on visual performance and eye fatigue. Hum Factor Ergon Man. 2002;12(2):193-209.

25. Perez-Carpinell J, Baldovi R, de Fez MD, Castro J. Color memory matching: Time effect and other factors. Color Res Appl. 1998;23(4):234-47.

26. Khairi AN, Falah MAF, Suyantohadi A, Takahashi N, Nishina H. Effect of Storage Temperatures on Color of Tomato Fruit (Solanum lycopersicum Mill.) Cultivated under Moderate Water Stress Treatment. Agric Agric Sci Proc. 2015;3:178-83.

27. Logvinenko AD. The geometric structure of color. J Vision. 2015;15(1).

28. Richardson AC, Boldingh HL, McAtee PA, Gunaseelan K, Luo ZW, Atkinson RG, David KM, Burdon JN, Schaffer RJ. Fruit development of the diploid kiwifruit, Actinidia chinensis 'Hort16A'. Bmc Plant Biol. $2011 ; 11$.

29. Lazier WW. Comptes rendu Proceedings of 1951 Stockholm Convention of the Commission Internationale De L'Eclairage. J Soc Motion Pict T. 1953;60(3):313-4.

30. Zeileis A, Hornik K, Murrell P. Escaping RGBland: Selecting colors for statistical graphics. Comput Stat Data An. 2009;53(9):3259-70.

31. Kendal D, Hauser CE, Garrard GE, Jellinek S, Giljohann KM, Moore JL. Quantifying Plant Colour and Colour Difference as Perceived by Humans Using Digital Images. Plos One. 2013;8(8).

32. Ngo GC, Macabebe EQB. Image Segmentation Using K-Means Color Quantization and DensityBased Spatial Clustering of Applications with Noise (DBSCAN) for Hotspot Detection in Photovoltaic Modules. Proceedings of the 2016 leee Region 10 Conference (Tencon). 2016:1614-8.

33. Huang ZK, Wang SQ, Hou LY. Segmentation of color textures using K-means cluster based wavelet image fusion. Information Technology for Manufacturing Systems, Pts 1 and 2. 2010:209-14.

34. Fullerton A, Keiding J. A comparison between a tristimulus colorimeter (Minolta ChromaMeter CR-200) and two spectrophotometers (Minolta Spectrophotometer CM-508i and CM-2002). Quantification of UV-B induced erythema in a hairless guinea pig model. Skin Res Technol. 1997;3(4):237-41.

35. Wang M, Li M, A. M. SELECTION OF A NEW RED-FLESHED KIWIFRUIT CULTIVAR 'HONGYANG'. Acta Horticulturae. 2003;610:115-7.

36. Gijsenij A, Gevers T, van de Weijer J. Computational Color Constancy: Survey and Experiments. leee T Image Process. 2011;20(9):2475-89.

37. Sharma G, Wu WC, Daa EN. The CIEDE2000 color-difference formula: Implementation notes, supplementary test data, and mathematical observations. Color Res Appl. 2005;30(1):21-30. 
38. Mokrzycki W, Tatol M. Color difference Delta E - A survey. Machine Graphics and Vision. 2011;20(4):383-411.

39. Witzel RF, Burnham RW, Onley JW. Threshold and Suprathreshold Perceptual Color Differences. J Opt Soc Am. 1973;63(5):615-25.

40. Bharathi PT, Subashini P. Texture Based Color Segmentation for Infrared River Ice Images Using K-Means Clustering. International Conference on Signal Processing, Image Processing and Pattern Recognition (Icsipr 2013). 2013:298-302.

41. Celebi ME. Improving the performance of k-means for color quantization. Image Vision Comput. 2011;29(4):260-71.

42. Wang NH, Zhang C, Bian SA, Chang PJ, Xuan LJ, Fan LJ, Yu Q, Liu ZG, Gu CH, Zhang SZ, Wang YL, Shen YM. Flavonoid Components of Different Color Magnolia Flowers and Their Relationship to Cultivar Selections. Hortscience. 2019;54(3):404-+.

43. Rubner Y, Tomasi C, Guibas LJ. The Earth Mover's Distance as a metric for image retrieval. Int J Comput Vision. 2000;40(2):99-121.

44. $\mathrm{Yu} \mathrm{ZH}, \mathrm{Herman} \mathrm{G}$. On the earth mover's distance as a histogram similarity metric for image retrieval. 2005 IEEE International Conference on Multimedia and Expo (ICME), Vols 1 and 2. 2005:686-9.

45. Takada K, Yanai K. Web Video Retrieval Based on the Earth Mover's Distance by Integrating Color, Motion and Sound. leee Image Proc. 2008:89-92.

46. Weller HI, Westneat MW. Quantitative color profiling of digital images with earth mover's distance using the R package colordistance. Peerj. 2019;7.

47. Murray JD, VanRyper W. Encyclopedia of graphics file formats: O'Reilly; 1996.

48. McCamy CS, Marcus H, Davidson JG. A Color Rendition Chart. Journal of Applied Photographic Engineering. 1976;11(3):95-9.

49. Richardson A, Eyre V, Kashuba P, Ellingham D, Jenkins H, Nardozza S. Early Shoot Development Affects Carbohydrate Supply and Fruit Quality of Red-Fleshed Actinidia chinensis var. chinensis 'Zes008'. Agronomy-Basel. 2021;11(1).

50. Hirst PM, D.S. T, I.J. W. Fruit colour responses of 'Granny Smith' apple to variable light environments. New Zealand Journal of Crop and Horticultural Science. 1990;18:205-14.

51. Alam J, Shaheen A, Anwar MS. Accessing select properties of the electron with ImageJ: an opensource image-processing paradigm. Eur J Phys. 2014;35(1).

52. Team RC. R: A Language and Environment for Statistical Computing. Vienna, Austria2020. 
53. Gama J, Davis G. colorscience: Color Science Methods and Data. R package version 1.0.8. 2019.

54. Pau G, Fuchs F, Sklyar O, Boutros M, Huber W. EBImage-an R package for image processing with applications to cellular phenotypes. Bioinformatics. 2010;26(7):979-81.

55. Gu ZG, Gu L, Eils R, Schlesner M, Brors B. circlize implements and enhances circular visualization in R. Bioinformatics. 2014;30(19):2811-2.

\section{Figures}

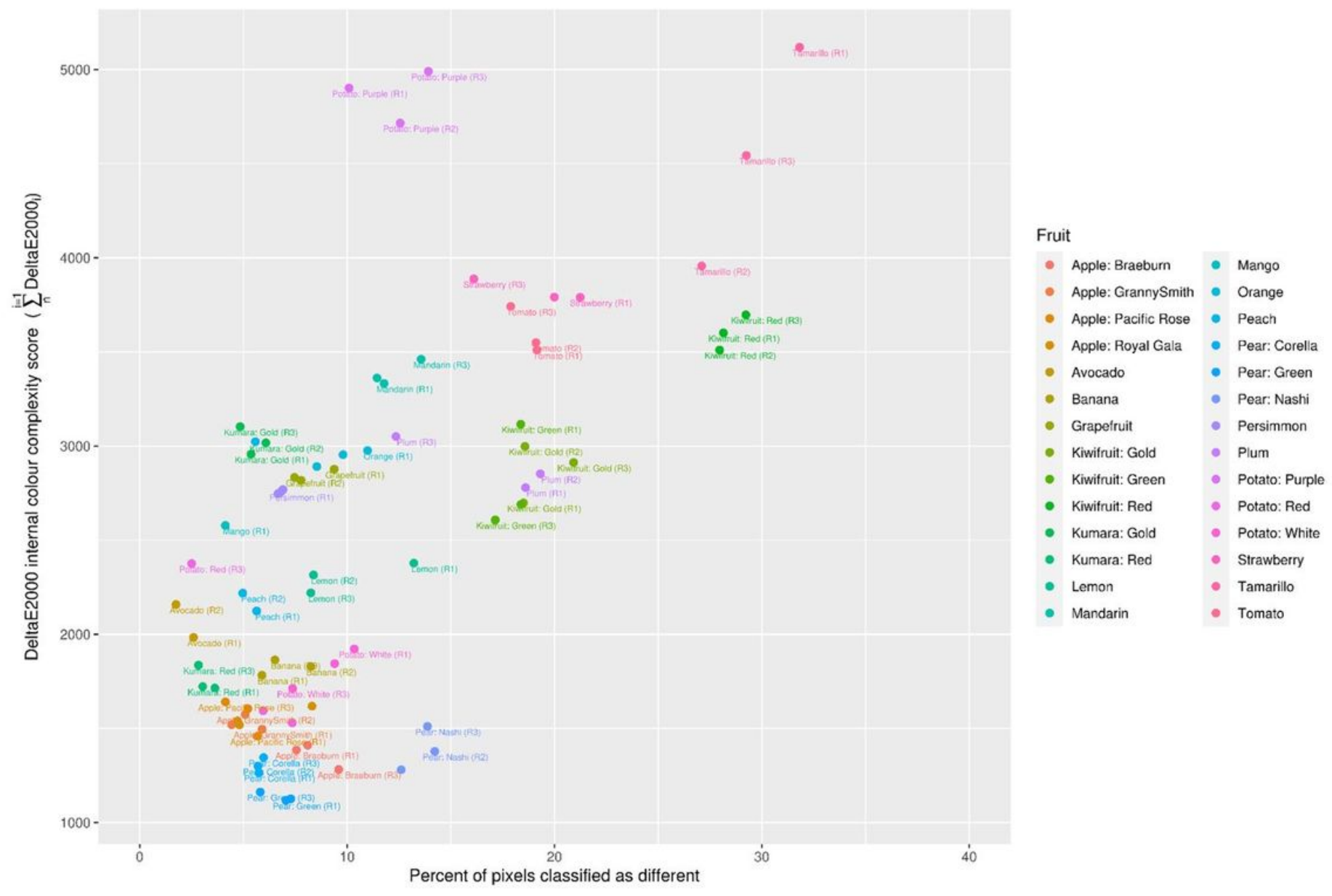

Figure 1

Classification of different colour values due to JPEG format compression compared with the colour complexity score of the fruit. $\mathrm{R}$, biological replicate. 


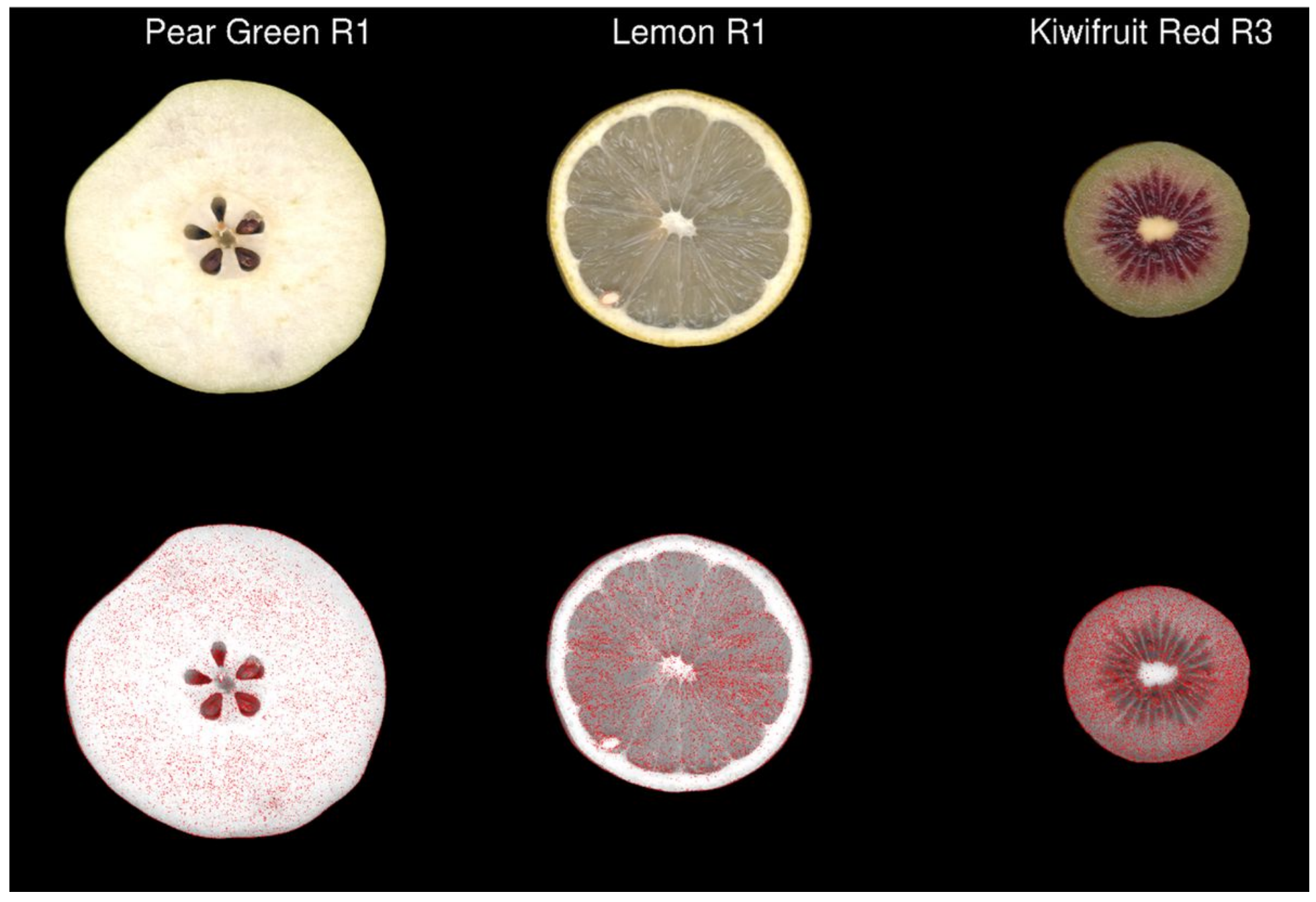

Figure 2

The 2-dimensional distribution of pixels classified as having different colour values in JPEG format compared with TIF format in three representative images of fruit. Pixels containing a $\Delta \mathrm{E} 2000$ distance greater than 2 between each format are coloured red in the second row. $\mathrm{R}$, biological replicate. 

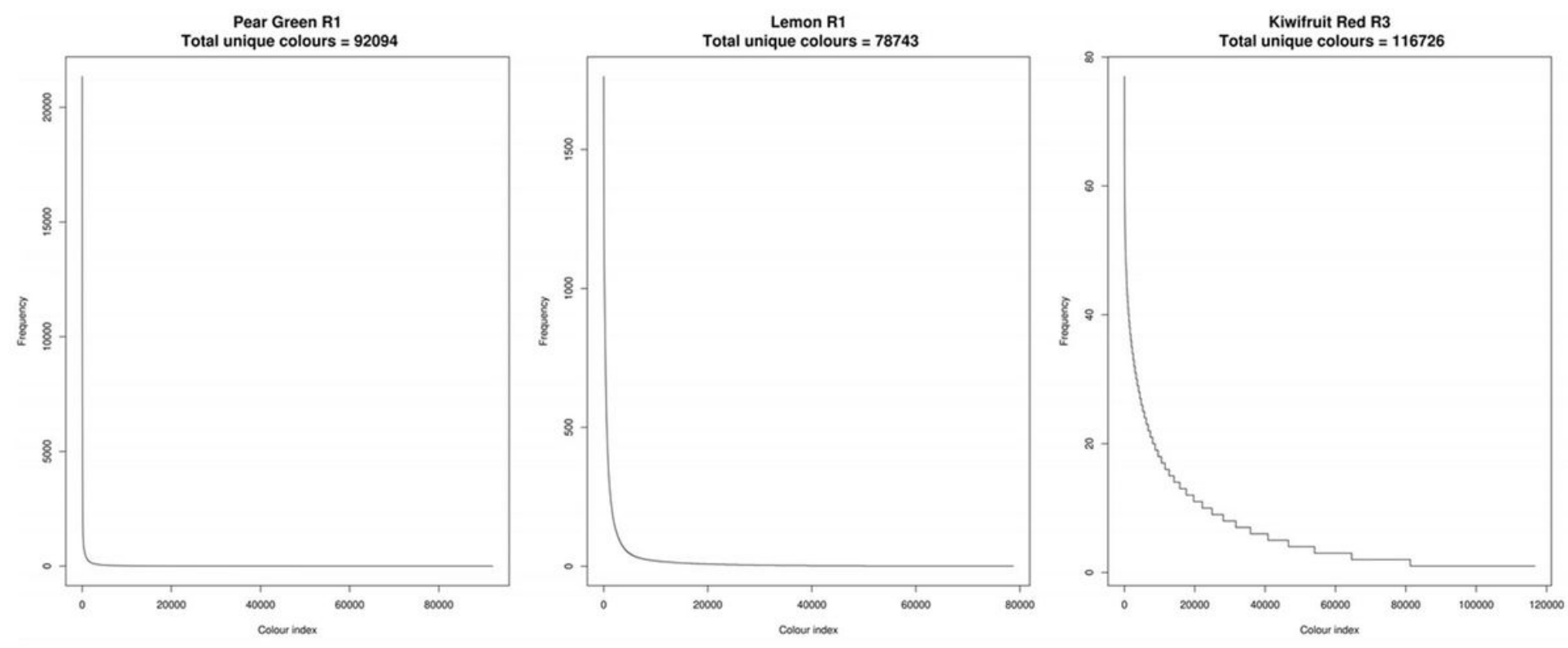

Figure 3

Ordered frequency distributions of unique colour values for three representative fruit. $\mathrm{R}$, biological replicate. 


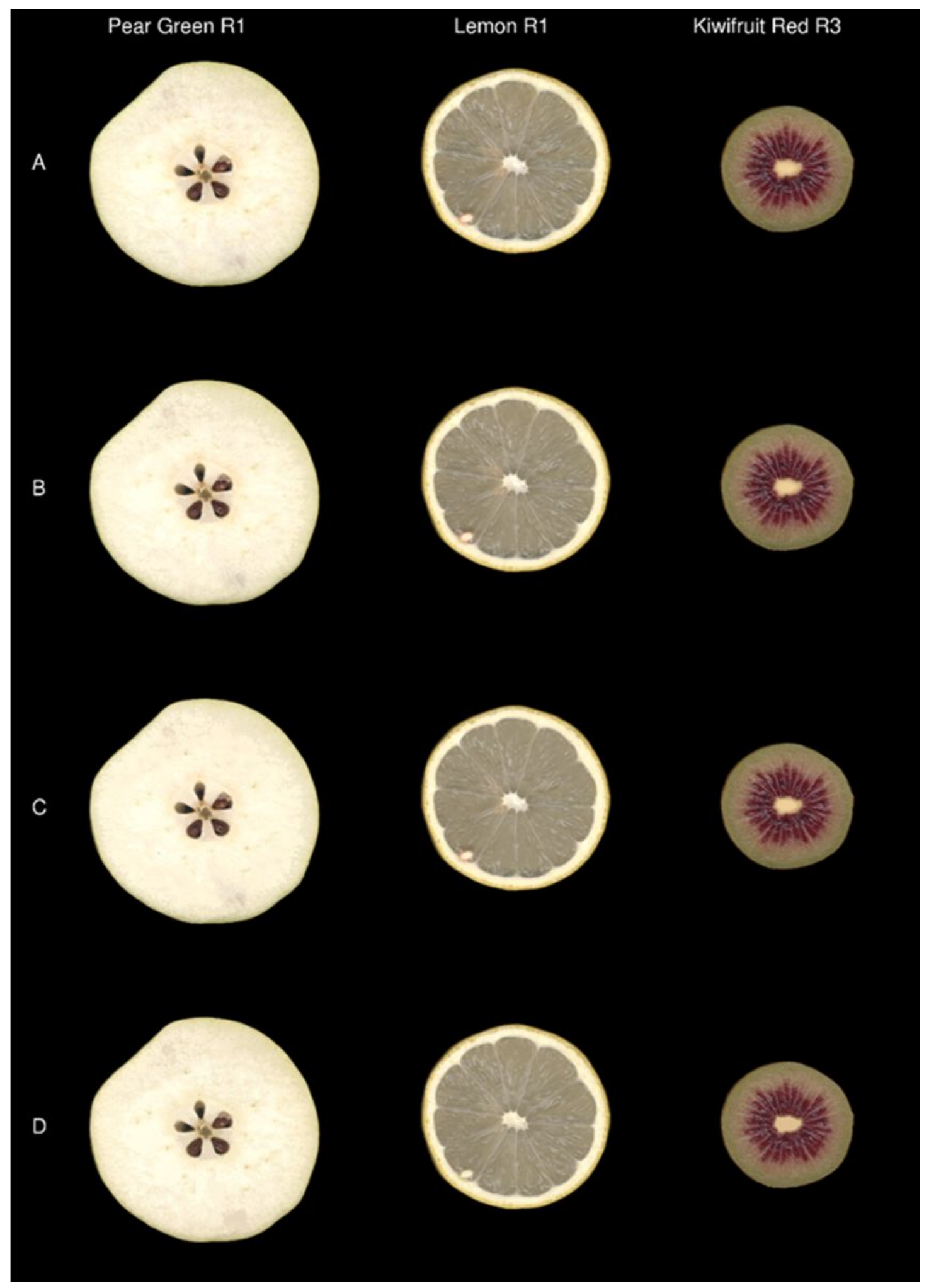

\section{Figure 4}

Original images (A) and simulated images of three representative fruit that were recoloured using the output colour bins generated by the region-growing algorithm with $\triangle E 2000$ at thresholds of 2 (B) and 3 (C) or by k-means clustering $(K=20)(D) R$, biological replicate. 


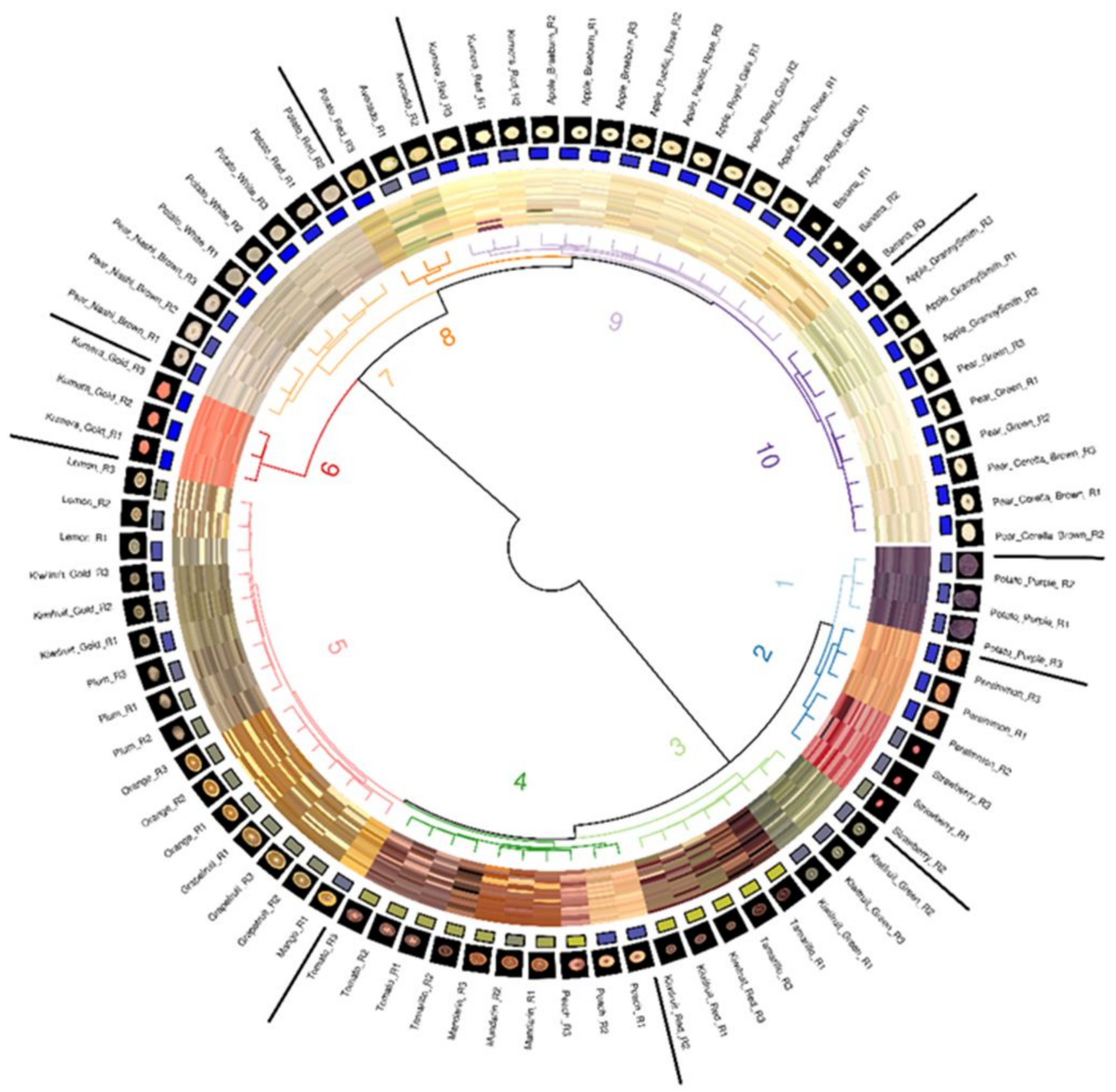

Track 1: Dendrogram height 8446.04

Track3: Cumulative percentage

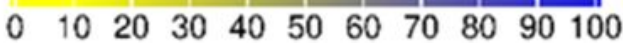

Figure 5

A circularized diagram displaying the clustering of fruit using the weighted $\Delta \mathrm{E} 2000$ distance metric. From the centre, track 1 shows a dendrogram of the clustered region grown data using the weighted $\Delta \mathrm{E} 2000$ distance metric against all colour values per fruit. Track 2 displays a visualisation matrix consisting of the top 20 colours summarised by the region-growing algorithm for each respective fruit. Track 3 shows 
the cumulative percentage of the top 20 colours for each respective fruit. Track 4 displays the raw image for each fruit. Track 5 indicates the name of each fruit. R, biological replicate.

A
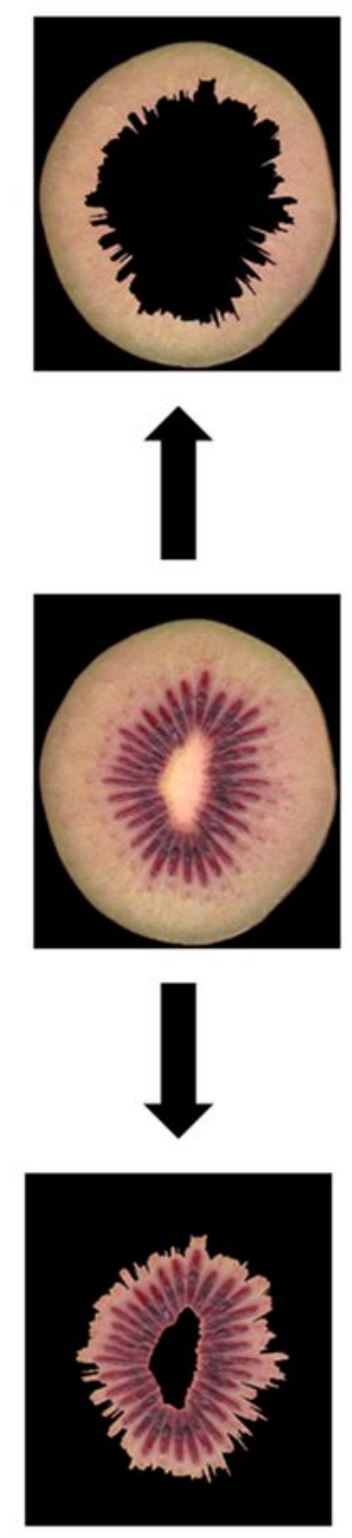
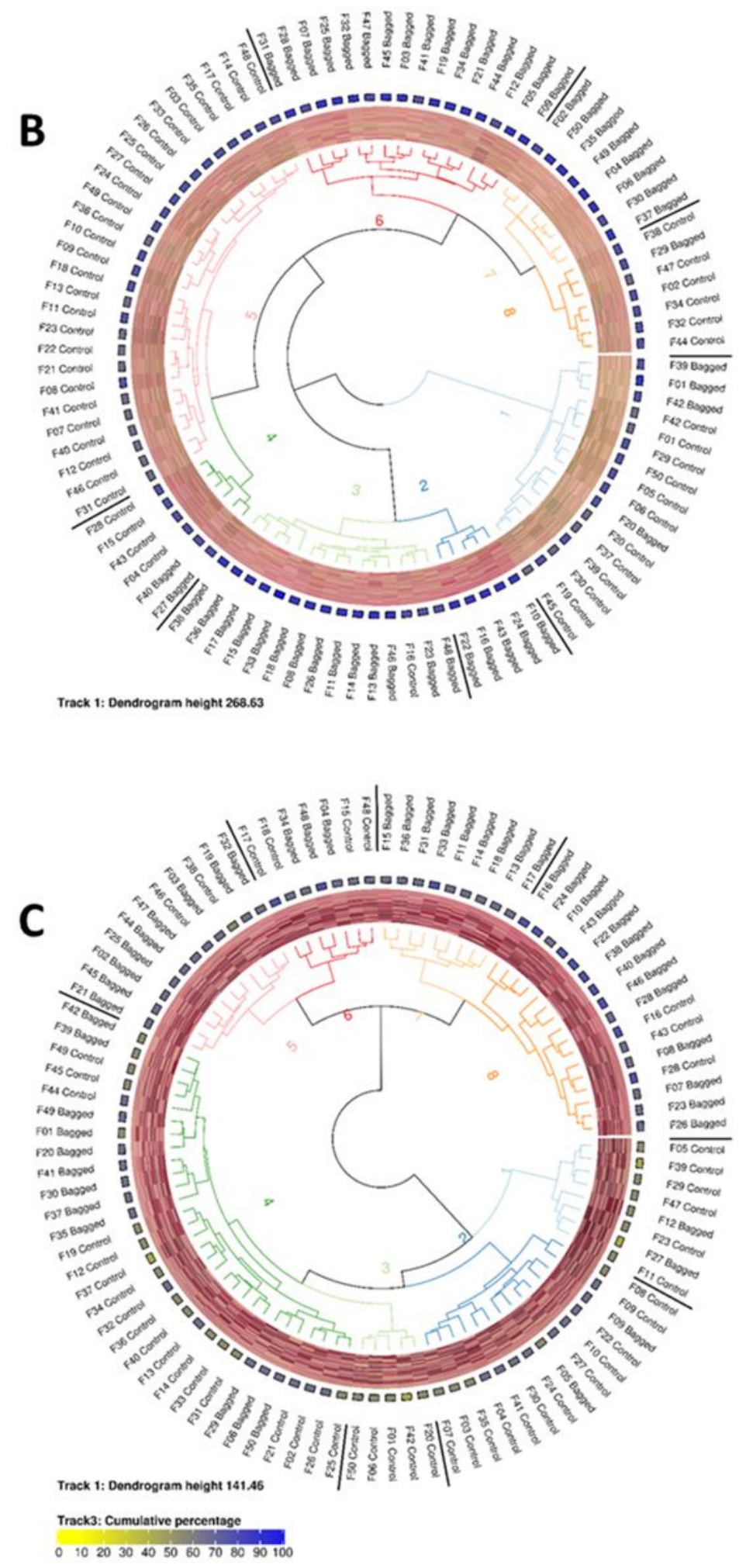

Figure 6

A) Representative images of segmented inner and outer pericarp tissues from Actinidia chinensis var. chinensis 'Zes008' kiwifruit. Fruit in this study were either treated with bagging or not (Control) to evaluate the effect of light exclusion on anthocyanin production (red colour accumulation) in fruit flesh. 
Circularised diagrams display the clustering of data from outer (B) and inner (C) pericarp segments using the weighted $\triangle \mathrm{E} 2000$ distance metric. From the centre, track 1 shows a dendrogram of the clustered region-grown data using the weighted $\Delta \mathrm{E} 2000$ distance metric against all colour values per fruit. Track 2 displays a visualisation matrix consisting of the top 20 colours summarised by the region-growing algorithm for each respective fruit. Track 3 shows the cumulative percentage of the top 20 colours for each respective fruit. Track 4 indicates the name of each fruit.

\section{Supplementary Files}

This is a list of supplementary files associated with this preprint. Click to download.

- Coverphoto.jpg

- Supplementaltable1.xlsx

- Supplementaltable2.xlsx

- SupplementaryFigure1.png 